\title{
Naso-pharyngeal sputum without a history of sinusitis as the first symptom of COVID-19 infection: a case report
}

\author{
fatemeh Taghizadeh ${ }^{1}$ and Hassan Taghizadeh ${ }^{2}$ \\ ${ }^{1}$ Affiliation not available \\ ${ }^{2}$ Shahid Beheshti University of Medical Sciences
}

April 28, 2020

\begin{abstract}
Naso-pharyngeal discharge as the first symptom of COVID-19 infection is presented in two cases . Appropriate diagnosis and isolation of the patients who may be at risk for covid-19 such as these cases help reduce further transmission.
\end{abstract}

Naso-pharyngeal sputum without a history of sinusitis as the first symptom of COVID-19 infection: a case report

\section{Key Clinical Message}

Naso-pharyngeal discharge as the first symptom of COVID-19 infection is presented in two cases . Appropriate diagnosis and isolation of the patients who may be at risk for covid-19 such as these cases help reduce further transmission.

Key words : COVID-19, Naso-pharyngeal sputum, sinusitis

\section{Introduction}

According to the World Health Organization (WHO) coronavirus disease 2019 (Covid-19) as a public health emergency is an international concern ${ }^{1}$, First identified in Wuhan, China, it can spread rapidly with a wide spectrum of severity ${ }^{2}$. There are various clinical features in COVID-19, ranging from asymptomatic state to multi organ dysfunction and acute respiratory distress syndrome ${ }^{3}$. The typical symptoms include cough, fever, headache, sore throat, myalgia, fatigue, breathlessness, and conjunctivitis in some cases ${ }^{4}$. Thus, it is hardly distinguishable from other respiratory infections. By the end of the first week, COVID-19 can lead to pneumonia, respiratory failure, and death ${ }^{5}$. About $66.6 \%$ of the patients had cough, but only $44.7 \%$ of whom had a fever. Also, the sputum production was observed in one-third of patients and sore throat was found in $14.0 \%$, Less than $5 \%$ of patients had gastrointestinal symptoms, such as nausea, diarrhea, and vomiting ${ }^{4}$. Fever and cough were the most common symptoms among patients with pneumonia caused by COVID- $19^{6}$.In one of the first reports on the disease, Huang et al. showed that the patients had dry cough, fever, dyspnea and malaise ${ }^{7}$. A suspect case is defined as a person with symptoms including cough sore throat and fever, who had travelled to China or other areas of local persistent transmission and had physical contacts with confirmed COVID-19 infection patients ${ }^{6}$. The cases may be asymptomatic or even without fever ${ }^{8,9}$. A confirmed case is a suspect case with a positive molecular test ${ }^{8}$. Nonetheless, we visited two cases in Modarres hospital clinic with naso-pharyngeal sputum who had no history of sinusitis as the first symptom.

We presented two patients infected with COVID-19 were followed with RT-PCR testing of throat swabs from Tehran, IRAN. 


\section{Case 1:}

The first case was a 45-year-old man who had a naso- pharyngeal discharge with no history of sinusitis for the past 6 days. When he went to the clinic, he had naso- pharyngeal discharge for two days, then he started to have a cough, myalgia, fatigue, fever and sweating. He had a positive RT-PCR result for COVID-19.

He was quarantined for 22 days with drug. Myalgia, fever and sweating decreased after 3 days, nasopharyngeal discharge, fatigue, and cough, continued after 28 days from onset of naso-pharyngeal discharge. He had no shortness of breath.

\section{Case 2:}

The second case was a 48-year-old woman with a naso-pharyngeal discharge without a history of sinusitis. After two days, she went to the clinic with chills, sweating, and burning in throat. She had no fever, cough, and shortness of breath and had a positive RT-PCR result for COVID-19. Having been quarantined for 15 days with drug, her chills and sweating were decreased, but she still felt burning in her throat after 29 days from onset of naso-pharyngeal discharge. The two patients were treated with antibiotic agents, antiviral agents, and supportive therapies at home.

\section{Discussion and Conclusion}

What makes the diagnosis of Covid-19 is that these patients do not have a fever or cough on the initial presentation ${ }^{10}$. Eventually, the report identifies the need to determine the full spectrum and natural history of clinical diseases, pathogenesis, and the duration of viral shedding related to the COVID-19 infection to inform the clinical management and public health decisions.If the findings in this report are replicated by a naso-pharengeal discharge as an asymptomatic carrier, it will be too demanding to prevent COVID-19 infection to help reduce further transmission The mechanism, by which carriers could acquire and transmit the coronavirus, requires supplementary investigation. Appropriate diagnosis and isolation of the patients who may be at risk for covid-19 such as these cases is important. Timely social distancing, diagnosis, and management may help reduce the mortality rates of COVID-19.

Conflict of Interest Disclosures : no conflict of interest

Author Contributions: FT: prepared this manuscript. HT: was the physician for these patient.

\section{References}

(1) Wang, D.; Hu, B.; Hu, C.; Zhu, F.; Liu, X.; Zhang, J.; Wang, B.; Xiang, H.; Cheng, Z.; Xiong, Y. Clinical characteristics of 138 hospitalized patients with 2019 novel coronavirus-infected pneumonia in Wuhan, China. Jama 2020 .

(2) Organization, W. H. Coronavirus disease (COVID-19) outbreak.URL https://www. who. int/emergencies/diseases/novelcoronavirus-2019 2020 .

(3) Zou, L.; Ruan, F.; Huang, M.; Liang, L.; Huang, H.; Hong, Z.; Yu, J.; Kang, M.; Song, Y.; Xia, J. SARSCoV-2 viral load in upper respiratory specimens of infected patients. New England Journal of Medicine 2020 .

(4) Lai, C.-C.; Liu, Y. H.; Wang, C.-Y.; Wang, Y.-H.; Hsueh, S.-C.; Yen, M.-Y.; Ko, W.-C.; Hsueh, P.-R. Asymptomatic carrier state, acute respiratory disease, and pneumonia due to severe acute respiratory syndrome coronavirus 2 (SARS-CoV-2): Facts and myths. Journal of Microbiology, Immunology and Infection 2020 .

(5) Singhal, T. A Review of Coronavirus Disease-2019 (COVID-19). The Indian Journal of Pediatrics 2020 , 87, 281-286.

(6) Lai, C.-C.; Shih, T.-P.; Ko, W.-C.; Tang, H.-J.; Hsueh, P.-R. Severe acute respiratory syndrome coronavirus 2 (SARS-CoV-2) and corona virus disease-2019 (COVID-19): the epidemic and the challenges. International journal of antimicrobial agents $2020,105924$. 
(7) Huang, C.; Wang, Y.; Li, X.; Ren, L.; Zhao, J.; Hu, Y.; Zhang, L.; Fan, G.; Xu, J.; Gu, X. Clinical features of patients infected with 2019 novel coronavirus in Wuhan, China. The Lancet 2020 ,395 , 497-506.

(8) Bai, Y.; Yao, L.; Wei, T.; Tian, F.; Jin, D.-Y.; Chen, L.; Wang, M. Presumed asymptomatic carrier transmission of COVID-19. Jama2020 .

(9) Cascella, M.; Rajnik, M.; Cuomo, A.; Dulebohn, S. C.; Di Napoli, R.: Features, evaluation and treatment coronavirus (COVID-19). InStatPearls [Internet] ; StatPearls Publishing, 2020.

(10) Guan, W.-j.; Ni, Z.-y.; Hu, Y.; Liang, W.-h.; Ou, C.-q.; He, J.-x.; Liu, L.; Shan, H.; Lei, C.-l.; Hui, D. S. C.; Du, B.; Li, L.-j.; Zeng, G.; Yuen, K.-Y.; Chen, R.-c.; Tang, C.-l.; Wang, T.; Chen, P.-y.; Xiang, J.; Li, S.-y.; Wang, J.-l.; Liang, Z.-j.; Peng, Y.-x.; Wei, L.; Liu, Y.; Hu, Y.-h.; Peng, P.; Wang, J.-m.; Liu, J.-y.; Chen, Z.; Li, G.; Zheng, Z.-j.; Qiu, S.-q.; Luo, J.; Ye, C.-j.; Zhu, S.-y.; Zhong, N.-s. Clinical Characteristics of Coronavirus Disease 2019 in China. New England Journal of Medicine 2020 . 